\title{
UNIFORMLY BOUNDED SET-VALUED COMPOSITION OPERATORS IN THE SPACES OF FUNCTIONS OF BOUNDED VARIATION IN THE SENSE OF WIENER
}

\author{
José Atilio Guerrero ${ }^{1}$, Janusz Matkowski ${ }^{2}$, Nelson Merentes ${ }^{3}$, Malgorzata Wróbel ${ }^{4}$ \\ ${ }^{I}$ Dpto. de Matemáticas y Física, Universidad Nacional Experimental del Táchira \\ San Cristóbal, Venezuela \\ ${ }^{2}$ Faculty of Mathematics, Computer Science and Econometrics, University of Zielona Góra \\ Zielona Góra, Poland \\ ${ }^{3}$ Escuela de Matemáticas, Universidad Central de Venezuela \\ Caracas, Venezuela \\ ${ }^{4}$ Institute of Mathematics and Computer Science, Jan Dtugosz University \\ Częstochowa, Poland \\ jaguerrero4@gmail.com,J.Matkowski@wmie.uz.zgora.pl,nmerucv@gmail.com \\ m.wrobel@ajd.czest.pl
}

\begin{abstract}
We show that the one-sided regularizations of the generator of any uniformly bounded set-valued Nemytskij composition operator mapping the space of bounded variation functions in the sense of Wiener into the space of bounded variation functions with closed bounded convex values (in the sense of Wiener) are affine functions.
\end{abstract}

Keywords: $\phi$-variation in the sense of Wiener, set-valued functions, left and right regularizations, uniformly bounded operator, composition (Nemytskij) operator, Jensen equation

\section{Introduction}

Let $(X,|\cdot|),\left(Y,|\cdot|_{Y}\right)$ be real normed spaces, $C$ be a convex cone in $X$ and $I=[a, b] \subset \mathbb{R}(a, b \in \mathbb{R}, \mathrm{a}<b)$ be an interval. Let $c l b(Y)$ denote the family of all non-empty bounded, closed and convex subsets of $Y$. For a given set-valued function $h: I \times C \rightarrow c l b(Y)$ we consider the composition (superposition) Nemytskij operator $H: C^{I} \rightarrow \operatorname{clb}(Y)^{I}$ defined by $H(F)=h(\cdot, F(\cdot))$ for $F \in C^{I}$. It is shown that if $H$ maps the space $B V_{\varphi}(I, C)$ of bounded $\varphi$-variation functions (in the sense of Wiener) into the space $B V_{\psi}(I, c l b(Y))$ of bounded $\psi$-variation functions with closed bounded convex values, and $H$ is uniformly bounded, then the one-sided regularizations $h^{-}$and $h^{+}$of $h$ with respect to the first variable exist and are affine with respect to the second variable. In particular,

$$
h^{-}(t, x)=A(t) x+{ }^{*} B(t), \quad t \in I, \quad x \in C,
$$


for some functions $A: I \rightarrow L(C, c l b(Y))$ and $B \in B V_{\psi}(I, c l b(Y))$, where $L(C, c l b(Y))$ stands for the space of all $*$ additive and continuous mappings acting from $C$ into $c l b(Y)$. This considerably extends the main result of the paper [1], where the uniform continuity of the operator $H$ is assumed.

Let us remark that the uniform boundedness of an operator (weaker than the usual boundedness), introduced and applied in [2] for the Nemytskij composition operators acting between spaces of Hölder functions in the single-valued case and then extended to the set-valued cases in [3] - for the operators with convex and compact values and in [4] - for the operators with convex and closed values.

\section{Preliminaries}

Let $\mathcal{F}$ be the set of all convex functions $\varphi:[0, \infty) \rightarrow[0, \infty)$ such that $\varphi(0)=$ $=0$ and $\varphi(t)>0$ for $t>0$.

Remark 2.1. [1. Remark 2.1]. If $\varphi \in \mathcal{F}$, then $\varphi$ is continuous and strictly increasing.

As usual, $X^{I}$ stands for the set of all functions $f: I \rightarrow X$.

Definition 2.2. Let $\varphi \in \mathcal{F}$ and $(X,|\cdot|)$ be a real normed space. A function $f \in X^{I}$ is of bounded $\varphi$-variation in the sense of Wiener in the interval $I$, if

$$
v_{\varphi}(f)=\sup _{\zeta} \sum_{i=1}^{m} \varphi\left(\left|f\left(t_{i}\right)-f\left(t_{i-1}\right)\right|\right)<\infty,
$$

where the supremum is taken over all finite and increasing sequences $\zeta=\left(t_{i}\right)_{i=0}^{m}$, $t_{i} \in I, m \in \mathbb{N}$. For $\varphi(t)=t^{p}(t \geq 0, p \geq 1)$ condition (1) coincides with the classical concept of variation in the sense of Jordan [5, Chapter 8] when $p=1$, and in the sense of Wiener [6] if $p>1$. The general Definition 2.2 was introduced by Young [7].

Denote by $B V_{\varphi}(I, X)$ the set of all functions $f \in X^{I}$ such that $v_{\varphi}(f \lambda)<\infty$ for some $\lambda>0$. It is known that $B V_{\varphi}(I, X)$ is a normed space endowed with the norm

$$
\|f\|_{\varphi}:=|f(a)|+p_{\varphi}(f), \quad f \in B V_{\varphi}(I ; X),
$$

where $p_{\varphi}: B V_{\varphi}(I, X) \rightarrow \mathbb{R}_{+}$is defined in the following way

$$
p_{\varphi}(f)=\inf \left\{\varepsilon>0: v_{\varphi}\left(\frac{f}{\varepsilon}\right) \leq 1\right\}
$$

For $X=\mathbb{R}$ the linear normed space $\left(B V_{\varphi}(I, \mathbb{R}),\|\cdot\|_{\varphi}\right)$ was studied by Musielak and Orlicz [8], Ciemnoczołowski and Orlicz [9] and Maligranda and Orlicz [10]. The functional $p_{\varphi}$ is called the Luxemburg-Nakano-Orlicz seminorm (see [11-13]). 
Let $\left(Y,|\cdot|_{Y}\right)$ be a real normed space. Denote by $c l b(Y)$ the family of all nonempty closed bounded convex subsets of $Y$.

Given $A, B \in \operatorname{clb}(Y)$, we put $A+B:=\{a+b: a \in A, b \in B\}$ and we introduce the operation ${ }^{*}$ in $c l b(Y)$ defined as follows:

$$
A+{ }^{*} B:=\operatorname{cl}(A+B),
$$

where $c l$ stands for the closure in $Y$. The class $\operatorname{clb}(Y)$ with the operation $+{ }^{*}$ is an Abelian semigroup, with $\{0\}$ as the zero element, which satisfies the cancellation law. Moreover, we can multiply elements of $c l b(Y)$ by nonnegative numbers and, for all $A, B \in \operatorname{clb}(Y)$ and $\lambda, \mu \geq 0$, the following conditions hold:

$$
1 \cdot A=A, \lambda(\mu A)=(\lambda \mu) A, \lambda\left(A+{ }^{*} B\right)=\lambda A+{ }^{*} \lambda B,(\lambda+\mu) A=\lambda A+{ }^{*} \mu A .
$$

Let $D$ stand for the Hausdorff metric on the space $\operatorname{clb}(Y)$, defined by the formula

$D(A, B):=\max \left\{\sup _{a \in A} \inf _{b \in B}|a-b|_{Y}, \sup _{b \in B} \inf _{a \in A}|a-b|_{Y}\right\}, \quad A, B \in \operatorname{clb}(Y)$.

It is easily seen that $(\operatorname{clb}(Y), D)$ is a metric space. It is complete, provided $(Y,|\cdot|)$ is a Banach space $[14$, p. 40$]$.

Let us recall [15, Lemma 2.2] that the Hausdorff metric $D$ is invariant with respect to translation, i.e.

$$
D\left(A+{ }^{*} B, A+{ }^{*} C\right)=D(A+B, A+C)=D(B, C) ; A, B, C \in \operatorname{clb}(Y),
$$

for all $A, B, C \in \operatorname{clb}(Y)$.

Definition 2.3. Let $\varphi \in \mathcal{F}$ and $F: I \rightarrow c l b(Y)$. We say that $F$ has bounded $\varphi$-variation in the Wiener sense if

$$
v_{\varphi}(F):=\sup _{\zeta} \sum_{i=1}^{m} \varphi\left(D\left(F\left(t_{i}\right), F\left(t_{i-1}\right)\right)\right)<\infty,
$$

where the supremum is taken over all finite and increasing sequences $\zeta=\left(t_{i}\right)_{i=0}^{m}$, $t_{i} \in I, m \in \mathbb{N}$.

Let

$$
B V_{\varphi}(I, \operatorname{clb}(Y)):=\left\{F \in \operatorname{clb}(Y)^{I}: v_{\varphi}(\lambda F)<\infty \text { for some } \lambda>0\right\}
$$

For $F_{1}, F_{2} \in B V_{\varphi}(I, c l b(Y))$ put

$$
D_{\varphi}\left(F_{1}, F_{2}\right):=D\left(F_{1}(a), F_{2}(a)\right)+p_{\varphi}\left(F_{1}, F_{2}\right)
$$


where

$$
p_{\varphi}\left(F_{1}, F_{2}\right)=\inf \left\{\varepsilon>0: V_{\varepsilon}\left(F_{1}, F_{2}\right) \leq 1\right\}
$$

and

$$
V_{\varepsilon}\left(F_{1}, F_{2}\right)=\sup _{\zeta} \sum_{i=1}^{m} \varphi\left(\frac{1}{\varepsilon} D\left(F_{1}\left(t_{i}\right)+{ }^{*} F_{2}\left(t_{i-1}\right) ; F_{2}\left(t_{i}\right)+{ }^{*} F_{1}\left(t_{i-1}\right)\right)\right)
$$

where the supremum is taken over all finite and increasing sequences $\zeta=\left(t_{i}\right)_{i=0}^{m}$, $t_{i} \in I, m \in \mathbb{N}$.

Now, let us quote the following two lemmas.

Lemma 2.4. [16, Theorem 1]. The set $B V_{\varphi}(I, c l b(Y))$ endowed with $D_{\varphi}$ is a metric space.

Lemma 2.5. [16, Lemma 6]. Let $F_{1}, F_{2} \in B V_{\varphi}(I, c l b(Y))$ and $\varphi \in \mathcal{F}$. Then, for $\lambda>0$,

$$
V_{\lambda}\left(F_{1}, F_{2}\right) \leq 1 \text { if and only if } p_{\varphi}\left(F_{1}, F_{2}\right) \leq \lambda
$$

Let $(X,|\cdot|),\left(Y,|\cdot|_{Y}\right)$ be two real normed spaces. A subset $C \subset Y$ is said to be a convex cone if $\lambda C \subset C$ for all $\lambda \geq 0$ and $C+C \subset C$. It is obvious that $0 \in C$. Given an arbitrary set-valued function $h: I \times C \rightarrow c l b(Y)$ we consider the composition operator $H: C^{I} \rightarrow c l b(Y)^{I}$ generated by $h$, i.e.,

$$
(H f)(t):=h(t, f(t)), f \in C^{I}, t \in I \text {. }
$$

A set-valued function $F: C \rightarrow \operatorname{clb}(Y)$ is said to be *additive, if

$$
F(x+y)=F(x)+{ }^{*} F(y)
$$

and *Jensen if

$$
2 F\left(\frac{x+y}{2}\right)=F(x)+{ }^{*} F(y)
$$

for all $x, y \in C$.

We will need the following

Lemma 2.6. [17, Corollary 4]. Let $C$ be a convex cone in a real linear space and let $\left(Y,|\cdot|_{Y}\right)$ be a Banach space. A set-valued function $F: C \rightarrow \operatorname{clb}(Y)$ is *Jensen if, and only if, there exists an *additive set-valued function $A: C \rightarrow \operatorname{clb}(Y)$ and a set $B \in \operatorname{clb}(Y)$ such that 


$$
F(x)=A(x)+{ }^{*} B
$$

for all $x \in C$.

Let $(X,|\cdot|),\left(Y,|\cdot|_{Y}\right)$ be real normed spaces and let $C \subset X$ be a convex cone. A set valued function $F: C \rightarrow c l b(Y)$ is said to be continuous, if it is continuous as a single-valued function from $C$ into the metric space $c l b(Y)$.

From now on, let the set $L(C, \operatorname{clb}(Y))$ consist of all set-valued functions $A: C \rightarrow$ $c l b(Y)$ which are *additive and continuous (so positively homogeneous), i.e.,

$$
\mathrm{L}(C, c l b(Y))=\left\{A \in c l b(Y)^{C}: A \text { is * additive and continuous }\right\} .
$$

\section{An auxiliary result and its consequences}

Let $\left(Y,|\cdot|_{Y}\right)$ be a Banach space, $I^{-}:=(a, b], \quad \psi \in \mathcal{F}$ and let $f \in B V_{\psi}(I, c l b(Y))$. A function $f^{-}: I^{-} \rightarrow c l b(Y)$ defined by

$$
f^{-}(t):=\lim _{s \uparrow t} f(s), \quad t \in I^{-},
$$

is called the left regularization of $f$. In what follows, denote by $B V_{\psi}{ }^{-}(I, c l b(Y)$ the subspace of $B V_{\psi}(I, c l b(Y))$ which consists of those functions that are left continuous on $(a, b]$.

Since the completeness of $\left(Y,|\cdot|_{Y}\right)$ implies the completeness of $\operatorname{clb}(Y)$ with respect to the Hausdorff metric, applying Lemma 10 from [16] with $(M, d)=(c l b(Y), D)$, we get the following lemma.

Lemma 3.1. If $\left(Y,|\cdot|_{Y}\right)$ is a Banach space and $f \in B V_{\psi}(I, \operatorname{clb}(Y))$ then

$$
f^{-} \in B V_{\psi}^{-}(I, \operatorname{clb}(Y)
$$

Thus, if $f \in B V_{\psi}(I, c l b(Y))$ then its left regularization is the left continuous function.

In the same way we can define the right regularization of $f$.

For a set $C \subset X$ put

$$
B V_{\varphi}(I, C):=\left\{f \in B V_{\varphi}(I, X): f(I) \subset C\right\}
$$

Theorem 3.2. Let $(X,|\cdot|)$ be a real normed space, $\left(Y,|\cdot|_{Y}\right)$ a real Banach space, $C \subset X$ a convex cone, $I \subset \mathbb{R}$ an arbitrary interval and let $\varphi, \psi \in \mathcal{F}$. Suppose that the set-valued function $h: I \times C \rightarrow c l b(Y)$ is such that, for any $t \in I$ the function $h(t, \cdot): C \rightarrow c l b(Y)$ is continuous with respect to the second variable. If the composition operator $H$ generated by the set-valued function $h$ maps $B V_{\varphi}(I, C)$ into $B V_{\psi}(I, c l b(Y))$ and satisfies the inequality 


$$
D_{\psi}\left(H\left(f_{1}\right), H\left(f_{2}\right)\right) \leq \gamma\left(\left\|f_{1}-f_{2}\right\|_{\varphi}\right), \quad f_{1}, f_{2} \in B V_{\varphi}(I, C),
$$

for some function $\gamma:[0, \infty) \rightarrow[0, \infty)$, then the left regularization of $h$, i.e. the function $h^{-}: I^{-} \times C \rightarrow \operatorname{clb}(Y)$ defined by

$$
h^{-}(t, x):=\lim _{s \uparrow t} h(s, x), \quad t \in I^{-}, \quad x \in C,
$$

exists and

$$
h^{-}(t, x)=A^{-}(t) x+{ }^{*} B^{-}(t), \quad t \in I^{-}, x \in C,
$$

for some function $A^{-}: I^{-} \rightarrow L(X, c l b(Y)), B^{-}: I^{-} \rightarrow \operatorname{clb}(Y) \quad$ and $B^{-} \in\left(B W_{\psi}(I, c l b(Y))\right.$.

Proof: For every $x \in C$, the constant function $f(t)=x, t \in I$, belongs to $B V_{\varphi}(I, C)$. Since $H$ maps $B V_{\varphi}(I, C)$ into $B V_{\psi}(I, \operatorname{clb}(Y))$, the function $H(f)=h(\cdot, x)$ belongs to $B V_{\psi}(I, c l b(Y))$. By Lemma 3.1, the completeness of $c l b(Y)$ with respect to the Hausdorff metric implies the existence of the left regularization $h^{-}$of $h$. Since $H$ satisfies (3), by the definition of the metric $D_{\psi}$, we obtain

$$
p_{\psi}\left(H\left(f_{1}\right), H\left(f_{2}\right)\right) \leq \gamma\left(\left\|f_{1}-f_{2}\right\|_{\varphi}\right), \quad f_{1}, f_{2} \in B V_{\varphi}(I, C) .
$$

From Lemma 2.5, if $\gamma\left(\left\|f_{1}-f_{2}\right\|_{\varphi}\right)>0$, the inequality (4) is equivalent to

$$
W_{\gamma\left(\left\|f_{1}-f_{2}\right\|_{\varphi}\right)}\left(H\left(f_{1}\right), H\left(f_{2}\right)\right) \leq 1, \quad f_{1}, f_{2} \in B V_{\varphi}(I, C) .
$$

Therefore, if

$$
a<s_{1}<t_{1}<s_{2}<t_{2}<\cdots<s_{m}<t_{m}<b, \quad s_{i}, t_{i} \in I, i \in\{1, \cdots, m\}, m \in \mathbb{N},
$$

the definitions of the operator $H$ and the functional $V_{\varepsilon}$ imply

$$
\sum_{i=1}^{m} \psi\left(\frac{D\left(h\left(t_{i}, f_{1}\left(t_{i}\right)\right)+{ }^{*} h\left(s_{i}, f_{2}\left(s_{i}\right)\right) ; h\left(t_{i}, f_{2}\left(t_{i}\right)\right)+{ }^{*} h\left(s_{i}, f_{1}\left(s_{i}\right)\right)\right)}{\gamma\left(\left\|f_{1}-f_{2}\right\| \varphi\right)}\right) \leq 1 .
$$

For $s^{\prime}, t^{\prime} \in \mathbb{R}, s^{\prime}<t^{\prime}$, we define the function $\eta_{s^{\prime}, t}: \mathbb{R} \rightarrow[0,1]$ by

$$
\eta_{s^{\prime}, t^{\prime}}(t):=\left\{\begin{array}{ccc}
0 & \text { if } & t \leq s^{\prime}, \\
\frac{t-s^{\prime}}{t^{\prime}-s^{\prime}} & \text { if } & s^{\prime} \leq t \leq t^{\prime}, \\
1 & \text { if } & t^{\prime} \leq t .
\end{array}\right.
$$

Let us fix $r \in I^{-}$. For arbitrary finite sequence $a<s_{1}<t_{1}<s_{2}<t_{2}<\cdots<$ $s_{m}<t_{m}<r$ and $x_{1}, x_{2} \in C, x_{1} \neq x_{2}$, the functions $f_{1}, f_{2}: I \rightarrow X$ defined by 


$$
\begin{gathered}
f_{1}(t):=\frac{1}{2}\left[\eta_{s_{i}, t_{i}}(t)\left(x_{1}-x_{2}\right)+x_{1}+x_{2}\right], t \in I, \\
f_{2}(t):=\frac{1}{2}\left[\eta_{s_{i}, t_{i}}(t)\left(x_{1}-x_{2}\right)+2 x_{2}\right], \quad t \in I
\end{gathered}
$$

belong to the space $B V_{\varphi}(I, C)$. From (6) and (7), we have

$$
f_{1}(\cdot)-f_{2}(\cdot)=\frac{x_{1}-x_{2}}{2}, \quad t \in I
$$

whence

$$
\left\|f_{1}-f_{2}\right\|_{\varphi}=\left|\frac{x_{1}-x_{2}}{2}\right|
$$

and moreover,

$$
f_{1}\left(t_{i}\right)=x_{1} ; \quad f_{2}\left(t_{i}\right)=\frac{x_{1}+x_{2}}{2} ; \quad f_{1}\left(s_{i}\right)=\frac{x_{1}+x_{2}}{2} ; \quad f_{2}\left(s_{i}\right)=x_{2} .
$$

Using (5), we hence get

$$
\sum_{i=1}^{m} \psi\left(\frac{D\left(h\left(t_{i}, x_{1}\right)+{ }^{*} h\left(s_{i}, x_{2}\right) ; h\left(t_{i}, \frac{x_{1}+x_{2}}{2}\right)+{ }^{*} h\left(s_{i}, \frac{x_{1}+x_{2}}{2}\right)\right)}{\gamma\left(\frac{\left|x_{1}-x_{2}\right|}{2}\right)}\right) \leq 1 .
$$

From the continuity of $\psi$ and the definition of $h^{-}$passing to the limit in (8) when $s_{1} \uparrow r$, we obtain that

$$
\sum_{i=1}^{m} \psi\left(\frac{D\left(h^{-}\left(r, x_{1}\right)+{ }^{*} h^{-}\left(r, x_{2}\right) ; 2 h^{-}\left(r, \frac{x_{1}+x_{2}}{2}\right)\right)}{\gamma\left(\frac{\left|x_{1}-x_{2}\right|}{2}\right)}\right) \leq 1,
$$

that is

$$
\psi\left(\frac{D\left(h^{-}\left(r, x_{1}\right)+{ }^{*} h^{-}\left(r, x_{2}\right) ; 2 h^{-}\left(r, \frac{x_{1}+x_{2}}{2}\right)\right)}{\gamma\left(\frac{\left|x_{1}-x_{2}\right|}{2}\right)}\right) \leq \frac{1}{m}
$$

Hence, since $m \in \mathbb{N}$ is arbitrary,

$$
\psi\left(\frac{D\left(h^{-}\left(r, x_{1}\right)+{ }^{*} h^{-}\left(r, x_{2}\right) ; 2 h^{-}\left(r, \frac{x_{1}+x_{2}}{2}\right)\right)}{\gamma\left(\frac{\left|x_{1}-x_{2}\right|}{2}\right)}\right)=0,
$$

and, as $\psi(z)=0$ only if $z=0$, we obtain

$$
D\left(h^{-}\left(r, x_{1}\right)+{ }^{*} h^{-}\left(r, x_{2}\right) ; 2 h^{-}\left(r, \frac{x_{1}+x_{2}}{2}\right)\right)=0 .
$$


Therefore

$$
2 h^{-}\left(r, \frac{x_{1}+x_{2}}{2}\right)=h^{-}\left(r, x_{1}\right)+{ }^{*} h^{-}\left(r, x_{2}\right)
$$

for all $r \in I^{-}$and $x_{1}, x_{2} \in C$.

Thus, for each $r \in I^{-}$, the function $h^{-}(r, \cdot)$ satisfies the *Jensen functional equation in $C$, and by Lemma 2.6, for every $t \in I^{-}$, there exists an *additive set-valued function $A^{-}(t): C \rightarrow c l b(Y)$ and a set $B^{-}(t) \in \operatorname{clb}(Y)$ such that

$$
h^{-}(t, x)=A^{-}(t) x+B^{-}(t) \text { for } x \in C, t \in I^{-}
$$

To show that $A^{-}(t)$ is continuous for any $t \in I^{-}$, let us fix $x, \bar{x} \in C$. By (2) and (9) we have

$$
D\left(A^{-}(t) x, A^{-}(t) \bar{x}\right)=D\left(A^{-}(t) x+{ }^{*} B^{-}(t)\right), \quad A^{-}(t) \bar{x}+{ }^{*} B^{-}(t)=D(h(t, x), h(t, \bar{x})) .
$$

Hence, the continuity of $h$ with respect to the second variable implies the continuity of $A^{-}(t)$ and, consequently, being *additive, $A(t) \in L(C, c l b(Y))$ for every $t \in I^{-}$. To prove that $B^{-} \in B V_{\psi}(I, c l b(Y))$ let us note that the *additivity of $A^{-}(t)$ implies $A^{-}(t) 0=\{0\}$. Therefore, putting $x=0$ in (8) we get $h^{-}(t, 0)=B^{-}(t)$, $t \in I^{-}$, which gives the required claim.

Remark 3.3. The representation of the right regularization $h^{+}$defined by

$$
h^{+}(t, x):=\lim _{s \downarrow t} h(s, x), \quad t \in I^{+}, x \in C,
$$

can be obtained in a similar way.

Remark 3.4. If the function $\gamma:[0, \infty) \rightarrow[0, \infty)$ is right continuous at 0 and $\gamma(0)=0$, then the assumption of the continuity of $h$ with respect to the second variable can be omitted, as it follows from [1].

Note that in the first part of Theorem 3.2 the function $\gamma:[0, \infty) \rightarrow[0, \infty)$ is completely arbitrary.

As an immediate corollary of Theorem 3.2 and Remark 3.3 we obtain the following corollary.

Corollary 3.5. Let $(X,|\cdot|)$ be a real normed space, $\left.\left(Y,|\cdot|_{Y}\right)\right)$ a real Banach space, $C$ a convex cone in $X$ and suppose that $\varphi, \psi \in \mathcal{F}$. If the composition operator $H$ generated by a set-valued function $h: I \times C \rightarrow \operatorname{clb}(Y)$ maps $B V_{\varphi}(I, C)$ into $B V_{\psi}(I, c l b(Y))$ and there exists a function $\gamma:[0, \infty) \rightarrow[0, \infty)$ right continuous at 0 with $\gamma(0)=0$ such that

$$
D_{\psi}\left(H\left(f_{1}\right), H\left(f_{2}\right)\right) \leq \gamma\left(\left\|f_{1}-f_{2}\right\|_{\varphi}\right), \quad f_{1}, f_{2} \in B V_{\varphi}(I, C),
$$


then

$$
\begin{gathered}
h^{-}(t, x)=h_{0}{ }^{-}(t) x+{ }^{*} h_{1}{ }^{-}(t), t \in I^{-}, x \in C, \text { and } \\
h^{+}(t, x)=h_{0}{ }^{+}(t) x+h_{1}{ }^{+}(t), t \in I^{+}, x \in C,
\end{gathered}
$$

for some $h_{0}{ }^{-}: I^{-} \rightarrow L(X, c l b(Y)), h_{0}{ }^{+}: I^{+} \rightarrow L(X, c l b(Y)), h_{1}{ }^{-}: I^{-} \rightarrow \operatorname{clb}(Y)$, and $h_{1}{ }^{+}: I^{+} \rightarrow \operatorname{clb}(Y)$.

\section{Uniformly bounded composition operator}

In this section the following definition plays a crucial role:

Definition 4.1. ([2], Definition 1) Let $X$ and $Y$ be two metric spaces. We say that a mapping $F: X \rightarrow Y$ is uniformly bounded if, for any $t>0$, there exists a real number $\gamma(t)$ such that for any nonempty set $B \subset X$, we have

$$
\operatorname{diam} B \leq t \Rightarrow \operatorname{diam} F(B) \leq \gamma(t)
$$

Remark 4.2. Obviously, every uniformly continuous operator or Lipschitzian operator is uniformly bounded. Note that, under the assumptions of this definition, every bounded operator is uniformly bounded.

The main result of this paper reads as follows:

Theorem 4.3. Let $(X,|\cdot|)$ be a real normed space, $(Y,|\cdot|)$ a real Banach space, $C \subset X$ a convex cone, $I \subset \mathbb{R}$ an arbitrary interval and let $\varphi, \psi \in \mathcal{F}$. Suppose that the set-valued function $h: I \times C \rightarrow c l b(Y)$ is such that, for any $t \in I$ the function $h(t, \cdot): C \rightarrow c l b(Y)$ is continuous with respect to the second variable. If the composition operator $H$ generated by a set-valued function $h: I \times C \rightarrow \operatorname{clb}(Y)$ maps $B V_{\varphi}(I, C)$ into $B V_{\psi}(I, c l b(Y))$ and is uniformly bounded, then

$$
\begin{array}{ll}
h^{-}(t, x)=A^{-}(t) x+B^{-}(t), & t \in I^{-}, x \in C, \\
h^{+}(t, x)=A^{+}(t) x+B^{+}(t), & t \in I^{+}, x \in C,
\end{array}
$$

for some functions $A^{-}: I^{-} \rightarrow L(X, c l b(Y)), A^{+}: I^{+} \rightarrow L(X, c l b(Y)), B^{-}: I^{-} \rightarrow$ $c l b(Y)$ and $B^{+}: I^{+} \rightarrow c l b(Y)$.

Proof: Take any $t \geq 0$ and arbitrary $f_{1}, f_{2} \in B V_{\varphi}(I, C)$ such that

$$
\left\|f_{1}-f_{2}\right\|_{\varphi} \leq t
$$

Since $\operatorname{diam}\left\{f_{1}, f_{2}\right\} \leq t$, by the uniform boundedness of $H$, we have

$$
\operatorname{diam} H\left(\left\{f_{1}, f_{2}\right\}\right) \leq \gamma(t),
$$


that is

$$
\left\|H\left(f_{1}\right)-H\left(f_{2}\right)\right\|_{\psi} \leq \operatorname{diam} H\left(\left\{f_{1}, f_{2}\right\}\right)=\gamma\left(\left\|f_{1}-f_{2}\right\|_{\varphi}\right)
$$

and the result follows from Theorem 3.2.

Remark 4.4. If the function $\gamma:[0, \infty) \rightarrow[0, \infty)$ in Definition 4.1 is right continuous at 0 and $\gamma(0)=0$ (or if only $\gamma\left(0^{+}\right)=0$ ), then, clearly, the uniform boundedness of the involved operator reduces to its uniform continuity.

It follows that Theorem 4.3 improves the results of [1], where $H$ is assumed to be uniformly continuous.

Recall that the representation of Lipschitz continuous Nemytskij operators acting in the spaces of functions of bounded variation was first considered in [18] and then in [19] and [16] (in the single-valued case), and in [20] in the set-valued case. Let us add that A. Smajdor and W. Smajdor [21], extending the single-valued result of [22], initiated an interesting and important study of the set-valued case.

\section{Conclusions}

We show that if a Nemytskij composition operator $H$ mapping the space of bounded variation functions, in the sense of Wiener, into another space of this type (including the set-valued case) is uniformly bounded, then the one-sided regularizations of its generator are affine functions. This significantly extends the result of [1] where $H$ maps $B V_{\varphi}(I, C)$ into the space $B V_{\psi}(I, C C(Y))$ with convex compact values and much stronger uniform continuity of $H$ is assumed.

\section{References}

[1] Azócar A., Guerrero J.A., Matkowski J., Merentes N., Uniformly continuous set-valued composition operators in the spaces of functions of bounded variation in the sense of Wiener, Opuscula Mathematica 2010, 30(1), 53-60.

[2] Matkowski J., Uniformly bounded composition operators between general Lipschitz function normed spaces, Topol. Methods. Nonlinear Aval. 2011, 38(2), 395-406.

[3] Matkowski J., Wróbel M., Uniformly bounded set-valued Nemytskij operators acting between generalized Hölder functions spaces, Cent. Eur. J. Math. 2012, 10(2), 609-618.

[4] Matkowski J., Wróbel M., Uniformly bounded Nemytskij operators generated by set-valued functions between generalized Hölder functions spaces, Discuss. Math. Differ. Incl. Control Optim. 2011, 31(2), 183-198.

[5] Natanson I.P., Theory of Functions of a Real Variable, Nauka, Moscow 1974 (in Russian).

[6] Wiener N., The quadratic variation of a function and its Fourier coefficients, Massachusetts J. Math. Phys. 1924, 3, 72-94.

[7] Young L.C., Sur une généralisation de la notion de variation de puissance piéme bornée au sens de N. Wiener, et sur la convergence des séries de Fourier, C.R. Acad. Sci. 1937, 204(7), 470-472.

[8] Musielak J., Orlicz W., On generalized variations (1), Studia Math. 1959, XVIII, 11-44. 
[9] Ciemnoczołowski J., Orlicz W., Composing functions of bounded $\varphi$-variation, Proc. Amer. Math. Soc. 1986, 96, 431-436.

[10] Maligranda L., Orlicz W., On some properties of functions of generalized variation, Monatsh. Math. 1987, 104, 53-60.

[11] Luxemburg W.A., Banach Function Spaces, Ph.D. Thesis, Technische Hogeschod te Deift, The Netherlands 1955.

[12] Nakano H., Modulared Semi - Ordered spaces, Tokyo 1950.

[13] Orlicz J.W., A note on modular spaces, I. Bull. Acad. Polon. Sci. Ser. Sci. Math. Astronom. Phys. 1961, 9, 157-162.

[14] Castaing C., Valadier M., Convex analysis and measurable multifunctions, Lecture Notes in Math. 1977, 580.

[15] De Blasi F.S., On differentiability of multifunctions, Pac. J. Math. 1976, 66, 67-81.

[16] Chistyakov V.V., Lipschitzian Nemytskii operators in the cones of mappings of bounded Wiener $\psi$-variation, Folia Math. 2004, 11(1), 15-39.

[17] Smajdor W., Note on Jensen and Pexider functional equations, Demonstratio Mathematica 1999, 32, 363-376.

[18] Matkowski J., Miś J., On a characterization of Lipschitzian operators of substitution in the space BV〈a,b〉, Math. Nachr. 1984, 117, 155-159.

[19] Matkowski J., Lipschitzian composition operators in some function spaces, Nonlinear Anal. 1997, 3, 719-726.

[20] Zawadzka G., On Lipschitzian operators of substitution in the space of set-valued functions of bounded variation, Radovi Matematicki 1990, 6, 279-293.

[21] Smajdor A., Smajdor W., Jensen equation and Nemytskij operator for set-valued functions, Rad. Mat. 1989, 5, 311-320.

[22] Matkowski J., Functional equations and Nemytskij operators, Funkc. Ekvacioj Ser. Int. 1982, $25,127-132$. 